\title{
Topological notions for Kauffman and Vogel's polynomial
}

\author{
Rui Pedro Carpentier \\ Departamento de Matemática and Centro de Matemática Aplicada \\ Instituto Superior Técnico \\ Avenida Rovisco Pais, 1049-001 Lisboa \\ Portugal
}

25rd July, 2001

\begin{abstract}
In 22 Kauffman and Vogel constructed a rigid vertex regular isotopy invariant for unoriented four-valent graphs embedded in three dimensional space. It assigns to each embedded graph $G$ a polynomial, denoted $[G]$, in three variables, $A, B$ and $a$, satisfying the skein relations:

$$
\begin{aligned}
& {[\aleph]=A[)(]+B[\asymp]+[\chi]} \\
& \left.[\curlyvee]=a[)], \quad[\wp]=a^{-1}[)\right]
\end{aligned}
$$

and is defined in terms of a state-sum and the Dubrovnik polynomial for links.

In 《4] it is proved, in the case $B=A^{-1}$ and $a=A$, that for a planar graph $G$ we have $[G]=2^{c-1}\left(-A-A^{-1}\right)^{v}$, where $c$ is the number of connected components of $G$ and $v$ is the number of vertices of $G$.

In this paper we will show how we can calculate the polynomial, with the variables $B=A^{-1}$ and $a=A$, without resorting to the skein relation.
\end{abstract}




\section{Introduction.}

In [1] a polynomial invariant is described for 4-valent rigid vertex embedded graphs. This polynomial, denoted $[G]$ for a graph $G$, with variables $A, B$ and $a$ satisfies the skein relation

$$
[\aleph]=A[)(]+B[\Longleftarrow]+[\Varangle]
$$

and restricted to a link gives the Dubrovnik polynomial with variables $a$ and $A-B$. It is proved in [2] that this polynomial is invariant for regular isotopies and satisfies the conditions

$$
\left.[`]=a[)],[\wp]=a^{-1}[)\right]
$$

This means that the polynomials of graph diagrams are the same if we can change one graph diagram to the other by the Reidemeister moves for 4-valent graphs except for the first move (see [1, 3]).

The non-invariance under the first Reidemeister move can be corrected using the twisting number of a graph. This is defined as the sum of the writhes over all knot-theoretic circuits of the graph. A knot-theoretic circuit of a graph is a closed walk on the graph which corresponds to a link component if we change all vertices into crossings, and its writhe is the sum of the signs of all its self-crossings. The twisting number of a graph is invariant under regular isotopies. Thus, defining $t(G)$ as the twisting number of a graph $G$, we have that $a^{-t(G)}[G]$ is invariant under any isotopy since

$$
t(\urcorner)=t())+1
$$

and

$$
t(\succ)=t())-1
$$

In [2] the following graphical calculus is proved.

Theorem 1 For 4-valent graph diagrams, differing only in the marked local picture, we have the following identities:

$$
\begin{aligned}
& [\supset 0]=\mu[)] \\
& [\varnothing]=\mathcal{O}[)]
\end{aligned}
$$




$$
\begin{aligned}
& {[\gamma]=(1-A B)[)(]+\gamma[\check{\smile}]-(A+B)[X]}
\end{aligned}
$$

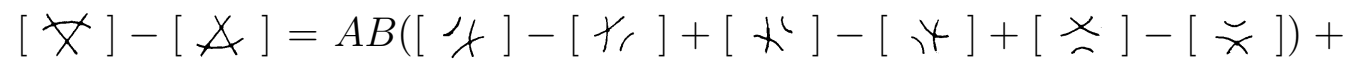

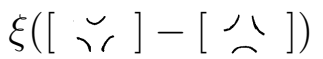

$\mu=\frac{a-a^{-1}}{A-B}+1$

$\mathcal{O}=\frac{A a^{-1}-B a}{A-B}-(A+B)$

$\gamma=\frac{B^{2} a-A^{2} a^{-1}}{A-B}+A B$

$\xi=\frac{B^{3} a-A^{3} a^{-1}}{A-B}$

As was shown in [4], this graphical calculus is sufficient to calculate the polynomial of a planar graph.

In [4] the author studied the case $B=A^{-1}$ and $a=A$. In this case, the graphical calculus takes the form:

$$
\begin{aligned}
& \text { [ ) } 0 \text { ] }=2[\text { ) ], } \\
& \left.[\varnothing]=-\left(A+A^{-1}\right)[)\right] \text {, } \\
& {[\gamma]=-\left(A+A^{-1}\right)[X] \text {, }}
\end{aligned}
$$

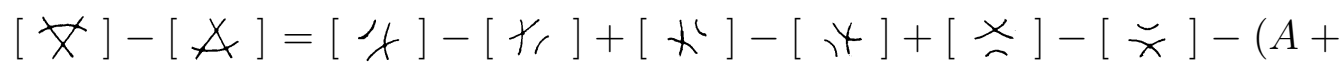

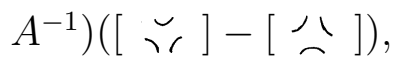

and for planar graphs we have:

Theorem 2 In the case $a=A$ and $B=A^{-1}$ for any 4-valent planar graph $G$ we have $[G]=2^{c-1}\left(-A-A^{-1}\right)^{v}$, where $c$ is the number of connected components of $G$ and $v$ is the number of vertices of $G$.

This means that a necessary condition for a 4 -valent rigid vertex embedded graph $G$ to be isotopic to a planar graph is that the polynomial of $G$ with $B=A^{-1}$ and $a=A$ is $[G]=2^{c-1}\left(-A-A^{-1}\right)^{v} A^{t(G)}$ where $c$ is the number of connected components, $v$ is the number of vertices and $t(G)$ is the 
twisting number of $G$. This corollary is a consequence of the fact that the polynomial $a^{-t(G)}[G]$ is an isotopy invariant.

Using the skein relation

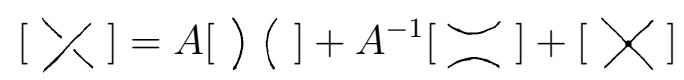

and the previous theorem it is easy to prove the following result:

Corollary 3 If a graph diagram $G$ has only one crossing, and the removal of this crossing does not change the number of connected components, then the polynomial of $G$ vanishes in the case $B=A^{-1}$ and $a=A$, and thus $G$ is not isotopic to a planar graph.

Thus we have a class of embedded graphs with polynomial equal to zero and therefore not isotopic to planar graphs. In the next section we will characterize the graphs with polynomial equal to zero.

\section{Calculating the polynomial for embedded graphs.}

Let a separating curve be a simple regular curve, on the plane, that intersects the diagram only at the crossings of the diagram and does so in the following way: $Y$ or $Y$. A partition of the diagram is a choice of marks of the type $X$ or $Y$, at each crossing, such that there exists a collection of disjoint separating curves intersecting all crossings of the diagram in agreement with these marks. A partition without marks we call a null partition日.

A graph diagram is called separable if it has a partition. We can show that this property is invariant under ambient isotopies. The planar graphs are clearly separable, since they have the null partition. Later we will see that any link is also separable.

Proposition 4 The polynomial, with variables $B=A^{-1}$ and $a=A$, of $a$ graph diagram is zero if and only if the graph diagram is not separable.

This result is a corollary of theorem 6 .

\footnotetext{
${ }^{1}$ This only exists for diagrams without crossings, i.e., planar graphs.
} 
There is a way to determine the partition of a graph. For that we need to introduce another definition.

A orientation on the edges of a graph will be called hyperbolic if at each vertex of the graph the orientation is of the type

Theorem 5 For a given graph diagram, we have that a choice of marks on the crossings is a partition if and only if there exists a hyperbolic orientation on the edges of the graph such that the mark on each crossing is in agreement with the orientation in the following way $Y$ or $\$.

In particular, we have that a graph is separable if and only if it has hyperbolic orientations.

Proof. Given a partition there exists a collection of separating curves that cross the graph diagram in agreement with the marks of the partition. These curves, together with the diagram, form the skeleton of a map that is bicolorable $\mathrm{e}^{2}$ since the vertices of the skeleton of the map have even degree (four in the case of the vertices of the diagram, and six in the case of the crossings of the diagram cut by the curves). Thus we can choose opposite orientations for the boundaries of the regions with different colors. This gives an orientation on the diagram that is constant on each edge (even when the edge through a crossing), is hyperbolic and such that at each crossing the separating curve cuts the diagram in the following way $Y$ or
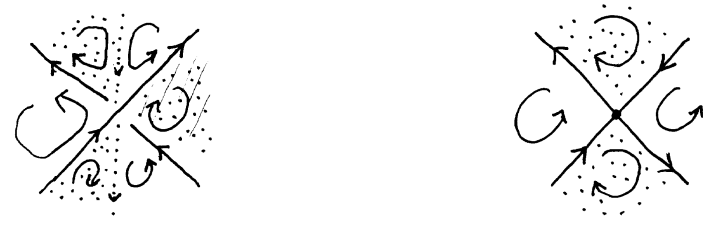

Given a hyperbolic orientation on the edges of the graph, we want to see that the marks $Y$ or $\$ form a partition. For that we take the regions delimited by the diagram. For each region the number of marks transversal to the boundary (i.e. such that the corresponding separating curve goes inside the region) is even since at each of this marks the orientation of the edges,

\footnotetext{
2i.e. the regions delimited by the diagram and the curves can be colored with two colors (black and white) in such way that two adjacent regions to the same edge of the diagram or part of the curve have different colors (see [5]).
} 
along the boundary, is reverse, whereas on the other crossings and vertices adjacent to the region the orientation is preserved since the orientation is hyperbolic (see fig.).

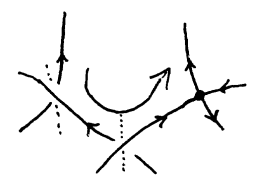

Thus, it is possible to join up such marks two by two using paths without intersections.

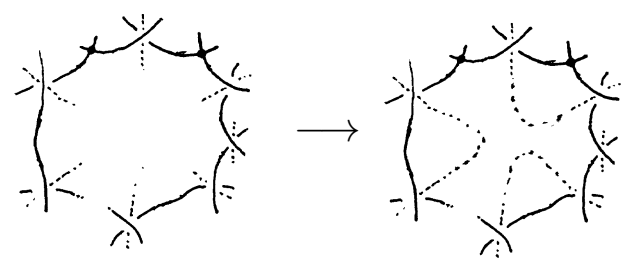

In the end we obtain a collection of separating curves for these marks, so we get a partition.

For a partition, we say that the crossing $Y$ has signature $A$ if its partition mark is vertical, and has signature $A^{-1}$ if its partition mark is horizontal. The product of the signatures of all crossings of the partition we call the signature of the partition. By convention we fix the value 1 for the signature of the null partition. For a graph diagram $G$ we denote by $\{G\}$ the sum of the signatures of all partitions of the diagram. If $G$ has no partition (it is non separable) we fix $\{G\}=0$.

Let $G$ be a graph diagram with a hyperbolic orientation on the edges, and let $w(G)$ be the writhe of $G$, that is, the sum of the signs of all crossings on this orientation. It is easy to see that the signature of the partition corresponding to the given orientation is $A^{w(D)}$.

A diagram component is a component corresponding to a connected component of the planar graph obtained by changing crossings into vertices. For example, a diagram of Hopf's link has only one diagram component although Hopf's link has two connected components.

We can also see that two hyperbolic orientations of the graph give us the same partition if and only if, on each diagram component, the orientations 
of the connected components of the graph are the same or oppositep'. Moreover, we have that, on each connected component of a graph, one hyperbolic orientation, if it exists, it is determined by the orientation of one edge in such a component.

Thus, calling $\mathcal{H}$ the set of all hyperbolic orientations of the diagram $G$, and $G^{h}$ the diagram $G$ with a given hyperbolic orientation $h$, we have

$$
\sum_{h \in \mathcal{H}} A^{w\left(G^{h}\right)}=2^{d}\{G\}
$$

where $d$ is the number of diagram components of $G$.

Theorem 6 The polynomial with variables $B=A^{-1}$ and $a=A$ for a graph diagram $G$ is

$$
\frac{1}{2}\left(-A-A^{-1}\right)^{v} \sum_{h \in \mathcal{H}} A^{w\left(G^{h}\right)}
$$

where $\mathcal{H}$ is the set of all hyperbolic orientations of the diagram $G, G^{h}$ is the diagram $G$ with a given hyperbolic orientation $h$ and $v$ is the number of vertices of the graph $G$.

Proof. We only need to check that the polynomial

$$
[G]=\frac{1}{2}\left(-A-A^{-1}\right)^{v} \sum_{h \in \mathcal{H}} A^{w\left(G^{h}\right)}
$$

satisfies the skein relation and that, for planar graphs, it is equal to $2^{c-1}(-A-$ $\left.A^{-1}\right)^{v}$, where $c$ is the number of connected components of $G$ and $v$ is the number of vertices of $G$.

First of all, we check the skein relation (with $B=A^{-1}$ and $a=A$ ).

Let $[X], \ldots,[)(], \ldots$ be the polynomials $[X],[)(], \ldots$ considering only the hyperbolic orientation with the local form $Y, \ldots),(, \ldots$.Then we have the following identities:

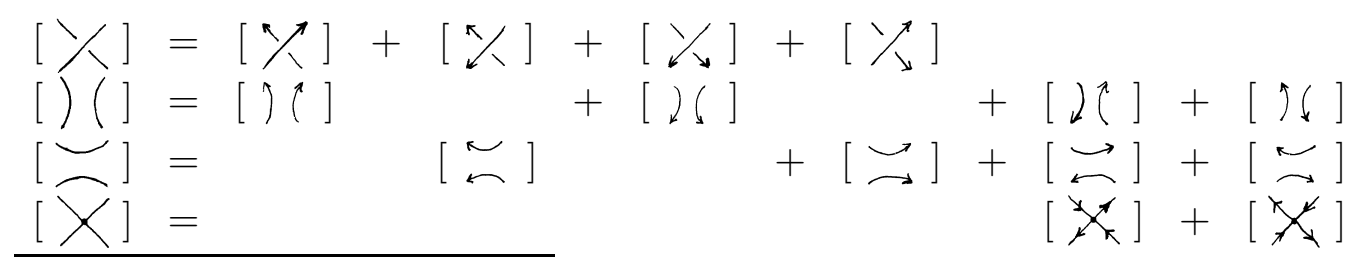

\footnotetext{
${ }^{3}$ This operation changes neither the writhe nor the hyperbolicity of the orientation.
} 
and

$$
\begin{aligned}
& {[\nu]=[\rightleftarrows]} \\
& {[16]=[\approx]} \\
& {\left[X_{k}\right]=-\left(A+A^{-1}\right)[2]} \\
& {[X]=-\left(A+A^{-1}\right)[96]} \\
& {[Y]=A[)(]} \\
& {[\ltimes]=A^{-1}[\stackrel{\swarrow}{\curvearrowleft}]}
\end{aligned}
$$

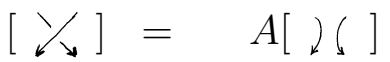

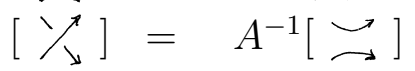

Thus we take:

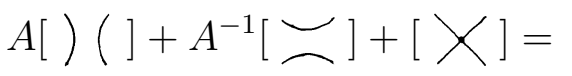

$$
\begin{aligned}
& =A([)(]+[2)]+[2(]+[) 6])
\end{aligned}
$$

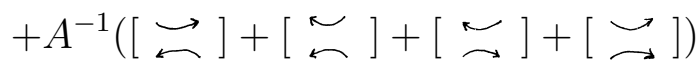

$$
\begin{aligned}
& +[\not x]+[\not X]=
\end{aligned}
$$

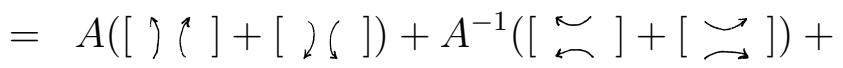

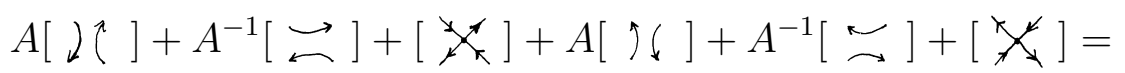

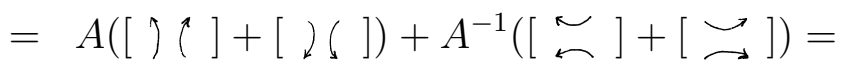

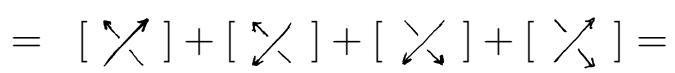

$$
\begin{aligned}
& =[\%]
\end{aligned}
$$

Since, for a planar graph $G$, the writhe of any hyperbolic orientation is zero, we have that $\sum_{h \in \mathcal{H}} A^{w\left(G^{h}\right)}$ is equal to the number of hyperbolic orientations which is $2^{c}$ where $c$ is the number of connected components of $G$. Thus the polynomial $[G]=\frac{1}{2}\left(-A-A^{-1}\right)^{v} \sum_{h \in \mathcal{H}} A^{w\left(G^{h}\right)}$ is equal to $2^{c-1}\left(-A-A^{-1}\right)^{v}$ for a planar graph $G$.

Since any orientation on a link is hyperbolic, we have that all links, including the knots, are separable.

In the case of the knots, we have only two orientations and these have the same writhe. Thus the polynomial for a knot $K$ is $[K]=A^{w(K)}$ where $w(K)$ is the writhe of $K$. In this way, the corrected polynomial $A^{-t(G)}[G]$ become trivial for knots. 
Another result that is easy to check is the following.

Corollary 7 If the graph admits a knot-theoretic circuit that passes through vertices an odd number of times then the graph is not separable.

This happens because a hyperbolic orientation reverses the orientations of the edges along a knot-theoretic circuit, whenever it passes through a vertex. This gives us a easy way to find non separable graphs (though it is not general).

\section{Conjectures.}

Now we consider the polynomial $P$ that for a graph $G$ gives the polynomial

$$
P(G)=\frac{A^{-t(G)}[G]}{2^{c-1}\left(-A-A^{-1}\right)^{v}} .
$$

This polynomial is invariant under isotopies and for planar graphs takes the value 1 . Moreover, this polynomial satisfies the following identities:

$$
\begin{aligned}
& P() \circ)=P(\quad) \text {; } \\
& P(\varnothing)=P()) \text {; } \\
& P(\gamma)=P()(\text { ), if } \gamma \text { and }) \text { ( admit hyperbolic orientations and )( } \\
& \text { has a hyperbolic orientation with the local form }) 6 \text { or } 2 \uparrow \text {; } \\
& P(\Varangle)=P(\not \Varangle) .
\end{aligned}
$$

We are interested in studying the class of the graphs with the polynomial $P$ equal to 1 (that is the class of graphs for which the polynomials $P$ or [ ] does not detect non-planarity). This class contains the planar graphs and the knots. Moreover, it contains any graph that can be obtained from a planar graph or a knot by the moves:

$$
\begin{aligned}
& 10 \rightleftharpoons) ; \\
& \varnothing \rightleftharpoons) ;
\end{aligned}
$$


$\gamma \rightleftharpoons)($, if $\gamma$ and $)($ are hyperbolic and $)($ has a hyperbolic orientation with the local form $) 6$ or $2 \pi$;

$\Varangle \rightleftharpoons A$.

Let $\mathcal{C}_{1}$ be the set of the graphs isotopic to planar graphs, $\mathcal{C}_{2}$ the set of the graphs isotopic to graphs obtained from planar graphs by these last moves, $\mathcal{C}_{3}$ the set of the graphs isotopic to graphs obtained from knotsf by these last moves, and $\mathcal{C}_{4}$ the set of the graphs with the polynomial $P$ equal to 1 .

We have that $\mathcal{C}_{1} \subseteq \mathcal{C}_{2}, \mathcal{C}_{2} \subseteq \mathcal{C}_{4}$ and $\mathcal{C}_{3} \subseteq \mathcal{C}_{4}$.

We have also that $\mathcal{C}_{2} \subseteq \mathcal{C}_{3}$ because, by the lemma 2 of 䧃, any 4-valent planar graph can be transformed into a disjoint union of circles (that are the knots theoretic circuits of the graph) by the moves:

$$
\begin{aligned}
& x \rightarrow 1 \\
& \gamma \rightarrow)(
\end{aligned}
$$

and

$$
\Varangle \rightarrow \not A
$$

and by the move

$$
\lcm{0} \rightarrow \text { ) }
$$

we obtain the unknot.

Question: Are any two of the sets $\mathcal{C}_{1}, \mathcal{C}_{2}, \mathcal{C}_{3}$ and $\mathcal{C}_{4}$ the same?

We have that $\mathcal{C}_{1} \neq \mathcal{C}_{2}$, as the following example shows:

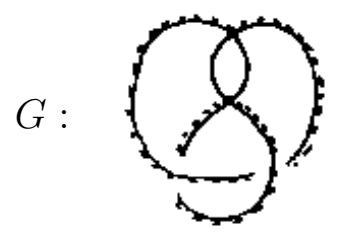

\footnotetext{
${ }^{4}$ including the unknot.
} 
Example 1 The graph in the picture is not planar, since it contains two disjoint linked cycles (indicated in the picture), but it can be obtained from a planar graph by the move $)(\rightarrow \gamma$.

Conjecture $8 \mathcal{C}_{2} \neq \mathcal{C}_{3}$

It is hard to believe that any knot can be obtained from a planar graph by the moves:

$$
\begin{aligned}
& \mathrm{\jmath O} \rightleftharpoons \mathrm{I} \\
& \varnothing \rightleftharpoons) \text {; } \\
& \gamma \rightleftharpoons)(\text {, if } \gamma \text { and })(\text { are hyperbolic and })(\text { has a hyperbolic } \\
& \not \rightleftharpoons \not .
\end{aligned}
$$

Conjecture $9 \mathcal{C}_{3} \neq \mathcal{C}_{4}$

It is possible that the following example belongs to $\mathcal{C}_{4}$ but not to $\mathcal{C}_{3}$.

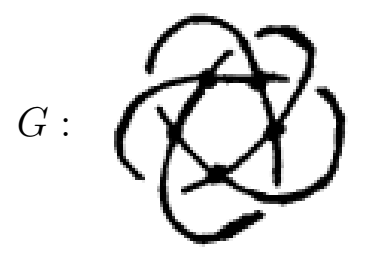

Example 2 The graph in the picture is a representation of the complete graph $K_{5}$ (thus is not planar), and has polynomial $P$ equal to 1.

Acknowledgment -I wish to thank Prof. Roger Picken who encouraged me to write this paper and helped me to improve it.

This work was supported by the program Programa Operacional "Ciência, Tecnologia, Inovação" (POCTI) of the Fundação para a Ciência e a Tecnologia (FCT), cofinanced by the European Community fund Feder. 


\section{References}

[1] L.H.Kauffman, Invariants of graphs in three-space, Trans. Amer. Math. Soc. 311, 2 (1989), 697-710.

[2] L.H.Kauffman and P.Vogel,Link polynomials and a graphical calculus, J. Knot Theory and Its Ramifications 1 (1992) 59-104.

[3] L.H.Kauffman, An invariant of regular isotopy, Trans. Amer. Math. Soc. 318, 2 (1990), 417-471.

[4] R.P.Carpentier, From planar graphs to embedded graphs - a new approach to Kauffman and Vogel's polynomial, J. Knot Theory and Its Ramifications 9(8) (2000) 975-986.

[5] T.L.Saaty and P.C.Kainen, The Four-Color Theorem: Assaults and Conquest, Dover Publications, New York, 1977.

[6] L.H.Kauffman, Knots and Physics, World Sci. Pub. (1991).

[7] S.Yamada, Invariants of spatial graphs, J. Graph Theory 13 (1989) 537551.

[8] A.Kawauchi, editor, A Survey of Knot Theory, Birkhauser (1996).

[9] V.V.Prasolov and A.B.Sossinsky, Knots, links, braids and 3-manifolds. An introduction to the new invariants in low-dimensional topology, Amer. Math. Soc., Providence, RI, 1996. 